\title{
Capillary and viscous fracturing during drainage in porous media
}

\author{
Francisco J. Carrillo $\odot^{*}$ \\ Department of Chemical and Biological Engineering, Princeton University, Princeton, New Jersey 08544, USA \\ Ian C. Bourg ${ }^{\dagger}$ \\ Department of Civil and Environmental Engineering, Princeton University, Princeton, New Jersey 08544, USA \\ and High Meadows Environmental Institute, Princeton University, Princeton, New Jersey 08544, USA
}

(Received 12 November 2020; revised 24 May 2021; accepted 24 May 2021; published 15 June 2021)

\begin{abstract}
Detailed understanding of the couplings between fluid flow and solid deformation in porous media is crucial for the development of novel technologies relating to a wide range of geological and biological processes. A particularly challenging phenomenon that emerges from these couplings is the transition from fluid invasion to fracturing during multiphase flow. Previous studies have shown that this transition is highly sensitive to fluid flow rate, capillarity, and the structural properties of the porous medium. However, a comprehensive characterization of the relevant fluid flow and material failure regimes does not exist. Here, we used our newly developed multiphase Darcy-Brinkman-Biot framework to examine the transition from drainage to material failure during viscously stable multiphase flow in soft porous media in a broad range of flow, wettability, and solid rheology conditions. We demonstrate the existence of three distinct material failure regimes controlled by nondimensional numbers that quantify the balance of viscous, capillary, and structural forces in the porous medium, in agreement with previous experiments and granular simulations. To the best of our knowledge, this study is the first to effectively decouple the effects of viscous and capillary forces on fracturing mechanics. Last, we examine the effects of consolidation or compaction on said dimensional numbers and the system's propensity to fracture.
\end{abstract}

DOI: 10.1103/PhysRevE.103.063106

\section{INTRODUCTION}

Multiphase flow in deformable porous media is a ubiquitous phenomenon in natural and engineered systems that underlies key processes in water and energy resource engineering and materials science, including membrane filtration, soil wetting/drying, enhanced hydrocarbon recovery, and geologic carbon sequestration [1-3]. A key obstacle to more accurate representations of this phenomenon is our limited understanding of the transition from fluid invasion to flowinduced fracturing, i.e., material failure caused by multiphase flow. In large part, this limitation is caused by a lack of computational approaches capable of representing multiphase flow in fractured deformable porous media.

Previous work on multiphase flow within static porous media is extensive and includes detailed examinations of the influence of wettability, viscosity, and flow rate on flow in unsaturated porous media at multiple scales. In particular, existing studies have demonstrated how capillary forces give rise to differences between drainage and imbibition [4]; how the ratio of fluid viscosities controls the stability of the invading fluid front [5-7]; and how the magnitude of the capillary

\footnotetext{
${ }^{*}$ https://github.com/Franjcf

'http://bourg.princeton.edu
}

Published by the American Physical Society under the terms of the Creative Commons Attribution 4.0 International license. Further distribution of this work must maintain attribution to the author(s) and the published article's title, journal citation, and DOI. number delineates distinct flow regimes [8,9]. Each of the aforementioned controls is highly dependent on the system of interest. This complicates efforts to develop general relative permeability and capillary pressure models that apply to most systems [10-13].

Flow of a single fluid phase through deformable porous media also has been studied in depth. Numerical modeling studies are largely based on the work of Biot and Terzaghi [14,15] and have been used to reproduce the behavior of arteries, boreholes, swelling clays, and gels [16-19]. Fundamental studies have generated detailed information on the dynamics that arise from fluid-solid couplings beyond the ideal poroelastic regime, including fracturing and cracking of granular and continuous systems [20-22]. In particular, these studies have shown that the main parameters controlling the deformation of a porous solid by single phase flow are the material softness and the magnitude of the fluid-solid momentum transfer.

The study of multiphase flow in a deformable porous medium is inherently more complex than the problems outlined above, as it requires simultaneous consideration of capillarity, wetting dynamics, fluid rheology, and solid deformation $[23,24]$. Deformation modes associated with material failure (i.e., multiphase fracturing) are particularly challenging as they require simultaneous representation of multiphase flow in fractures and in the surrounding porous matrix. Existing detailed examinations of this phenomenon have focused exclusively on granular systems [25-27]. Notably, Holtzman and Juanes [28,29] used experiments and discrete element models to demonstrate that the transitions between capillary fingering, viscous fingering, and fracturing during multiphase flow in granular media reflect two nondimensional numbers: 
a fracturing number (ratio of fluid driving force to solid cohesive force) and a modified capillary number (the ratio between viscous and capillary pressure drops). Other discrete element approaches have shown that fracturing is highly dependent on the invading fluid's capillary entry pressure [30,31].

To date, no study has yet examined how these conclusions translate to non-granular systems or to systems exhibiting viscously-stable drainage. Most studies have neglected this flow regime in favor of the more-common viscously-stable imbibition or viscously unstable drainage regimes. This observation is significant because naturally-occurring porous media often are highly heterogeneous and are (or eventually become) mixed-wet [32-35]. As such, all the associated flow regimes need to be investigated if we are to gain an adequate and complete understanding of multiphase flow in porous media.

In addition, to the best of our knowledge, no experimental or numerical investigation has simultaneously explored and decoupled the effects that flow rate, wettability, and deformability have on fracturing mechanics and/or identified the controlling parameters that relate all three properties within a single phase diagram. This is partially due to the fact that the associated parameter space is very large. Here, we explore a portion of this space using simulations carried out with our new multiphase Darcy-Brinkman-Biot (DBB) framework [36] with the goal of gaining insight into the nondimensional parameters that govern fracturing in deformable porous media. We also find that the fracturing dynamics predicted by our continuum-scale framework are consistent with those observed by the aforementioned granular studies. This suggests that, for ductile materials such as those represented here, a volume-averaged representation may be sufficient to capture the onset and propagation of fractures at the continuum scale.

\section{MODELING FRAMEWORK}

Our investigation is carried out through the use of the Multiphase DBB modeling framework, a new and flexible model used to simulate incompressible two-phase flow through and around deformable porous media [36]. It consists of five volume averaged fluid and solid conservation equations that are coupled by spatially dependent momentum exchange and capillary force terms. The model is composed of a fluid mass conservation equation,

$$
\frac{\partial \phi_{f}}{\partial t}+\nabla \cdot \boldsymbol{U}_{f}=0
$$

a fluid saturation conservation equation,

$$
\frac{\partial \phi_{f} \alpha_{w}}{\partial t}+\nabla \cdot\left(\alpha_{w} \boldsymbol{U}_{f}\right)+\nabla \cdot\left(\phi_{f} \alpha_{w} \alpha_{n} \boldsymbol{U}_{r}\right)=0,
$$

a fluid momentum conservation equation,

$$
\begin{aligned}
& \frac{\partial \rho_{f} \boldsymbol{U}_{f}}{\partial t}+\nabla \cdot\left(\frac{\rho_{f}}{\phi_{f}} \boldsymbol{U}_{f} \boldsymbol{U}_{f}\right) \\
& =-\phi_{f} \boldsymbol{\nabla} p+\phi_{f} \rho_{f} \boldsymbol{g}+\nabla \cdot \boldsymbol{S}-\phi_{f} \mu k^{-1}\left(\boldsymbol{U}_{f}-\boldsymbol{U}_{s}\right) \\
& \quad+\phi_{f} \boldsymbol{F}_{c}-\phi_{f} p_{c} \boldsymbol{\nabla} \alpha_{w},
\end{aligned}
$$

a solid mass conservation equation,

$$
\frac{\partial \phi_{s}}{\partial t}+\nabla \cdot\left(\phi_{s} \boldsymbol{U}_{s}\right)=0
$$

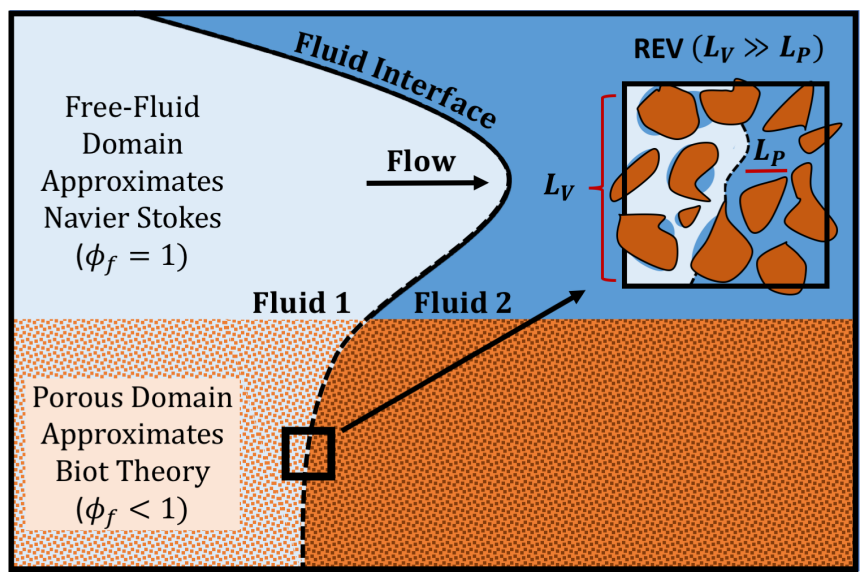

FIG. 1. Conceptual representation of the Multiphase DBB framework. The porous domain is shown in the lower half, the free-fluid domain is shown in the upper half, the two immiscible fluids (left and right) are shown in different shades of blue and are separated by an interface (black), and $\phi_{f}$ is the porosity. REV is the "representative elementary volume" over which all equations are averaged.

and a solid momentum conservation equation,

$$
\begin{aligned}
-\nabla \cdot \sigma= & -\phi_{s} \nabla p+\phi_{s} \rho_{s} \boldsymbol{g} \\
& +\phi_{f} \mu k^{-1}\left(\boldsymbol{U}_{f}-\boldsymbol{U}_{s}\right)-\phi_{f} \boldsymbol{F}_{c}-\phi_{s} p_{c} \nabla \alpha_{w} .
\end{aligned}
$$

In the previous equations, $\phi_{f}$ is the fluid volume fraction, $\phi_{s}$ is the solid volume fraction, $\alpha_{w}$ is the wetting fluid saturation, $\alpha_{n}$ is the nonwetting fluid saturation, $\boldsymbol{U}_{f}$ is the single-field fluid velocity, $\boldsymbol{U}_{s}$ is the solid velocity, $\boldsymbol{U}_{r}$ is the relative velocity of the two immiscible fluids, $p$ is the singlefield fluid pressure, $S$ is the volume averaged fluid viscous stress tensor, $\sigma$ is the volume averaged solid stress tensor, $\mu k^{-1}$ is the drag coefficient (a function of permeability $k$ and single-field fluid viscosity $\mu), \rho_{s}$ is the solid density, $\boldsymbol{g}$ is gravity, $p_{c}$ is the average capillary pressure in the porous medium (equal to zero in the solid-free region), and $\boldsymbol{F}_{c}$ represents additional capillary terms. Here, "single-field" refers to averaged variables that depend on the properties of both fluids. Last, $\rho_{f}=\alpha_{w} \rho_{w}+\alpha_{n} \rho_{n}$ and $\mu=\alpha_{w} \mu_{w}+\mu_{n} \rho_{n}$ are the single-field fluid density and viscosity, respectively. The closed form representations for $\boldsymbol{U}_{r}, \mu k^{-1}$, and $\boldsymbol{F}_{c}$ can be found in Appendix A.

As indicated in Fig. 1, the system of equations presented above asymptotically approaches the Navier-Stokes multiphase volume-of-fluid [37] equations in solid free regions (where $\phi_{f}=1, k$ is very large, and viscous drag is negligible) and multiphase Biot theory in porous regions (where $\phi_{f}<1$, $k$ is small, $\operatorname{Re}<1$, and drag dominates). This last point can be demonstrated by adding Eqs. (3) and (5) together within the porous domain, which results in the main governing equation used in multiphase Biot theory [36,38,39]:

$$
\nabla \cdot \sigma=\nabla p-\left(\phi_{s} \rho_{s}+\phi_{f} \rho_{f}\right) \boldsymbol{g}+p_{c} \nabla \alpha_{w} .
$$

A thorough discussion, derivation, and validation of this model can be found in Carrillo and Bourg [36] and related publications $[18,40]$. The two major limitation of the framework highlighted in these previous studies are as follows. 


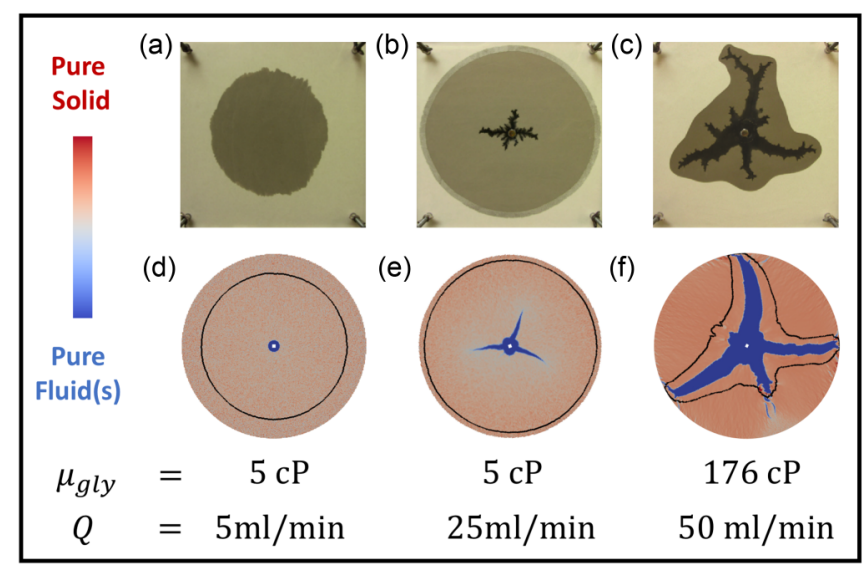

FIG. 2. Continuous transition from fluid imbibition to fracturing in a Hele-Shaw cell. Experimental images $(\mathrm{a}-\mathrm{c})$ were taken from Huang et al. [43] and numerically replicated using equivalent conditions (D-F). Black lines represent the advancing saturation front. Additional cases can be found in Ref. [36].

First, there needs to be a clear length-scale separation between the averaging volume, the sub-REV heterogeneities, and the overall system [41]. This condition is sustained in most situations involving fractured porous materials, where fracture width is generally significantly larger than the pore width within the porous matrix, with the possible exception of microfractures. Second, closure of the system of equations necessitates the use of parametric models describing the average behavior of the capillary pressure, permeability, and solid rheology within porous domains. As such, the accuracy of the overall model is inherently sensitive to the limitations and assumptions of these parametric models. The complete numerical implementation of the solver, its validations, and the cases shown within this study can be found within the open-source simulation package "hybridBiotInterFoam" [42].

\section{NUMERICAL SIMULATIONS}

\section{A. Crossover from imbibition to fracturing in a Hele-Shaw cell}

In addition to the derivation and extensive quantitative validation of Eqs. (1)-(5), our recent work [36] included a qualitative validation of the ability of the Multiphase DBB model to predict the transition from invasion to fracturing during multiphase flow. Briefly, this validation replicated experiments by Huang et al. [43] involving the injection of aqueous glycerin into dry sand at incremental flow rates within a 30 by 30 by $2.5 \mathrm{~cm}$ Hele-Shaw cell. As shown in Fig. 2, these experiments are inherently multiphysics, as fluid flow is governed by Stokes flow in the fracture (aperture $\sim \mathrm{cm}$ ) and by multiphase Biot theory in the porous sand (pore width $\sim 100 \mu \mathrm{m})$.

As discussed in our previous work, the similarities between our model and the experimental results are evident: As the viscous forces imposed on the solid increase, so does the system's propensity to exhibit fracturing as the primary flow mechanism (as opposed to imbibition). Minor microstructural differences between our simulations and the experiments reflect the manner in which the implemented continuum-scale rheology model approximates the solid's granular nature. It is clear, however, that both systems are controlled by the balance between viscous forces and solid rheology at the scale of interest [36]. As such, these simulations present an ideal starting point for our investigation.

\section{B. Creation of fracturing phase diagrams}

Here, we use the same simulation methodology developed in Ref. [36] and illustrated in Fig. 2 to gain insight into the general nondimensional parameters that control the observed transitional behavior between invasion and fracturing in a plastic porous medium. To do so, we systematically vary the solid's porosity ( $\phi_{f}=0.4$ to 0.8$)$, density-normalized plastic yield stress $\left(\tau_{\text {yield }}=1.5\right.$ to $24 \mathrm{~m}^{2} / \mathrm{s}^{2}$ ), capillary entry pressure $\left(p_{c, 0}=100\right.$ to $\left.50,000 \mathrm{~Pa}\right)$, and permeability $\left(k=1 \times 10^{-13}\right.$ and $\left.5 \times 10^{-9} \mathrm{~m}^{2}\right)$ as well as the invading fluid's viscosity $\left(\mu_{n}=0.5\right.$ to $\left.50 \mathrm{cP}\right)$ and injection rate $\left(\boldsymbol{U}_{f}=\right.$ $1 \times 10^{-4}$ to $\left.8 \times 10^{-2} \mathrm{~m} / \mathrm{s}\right)$. As in our previous work, the solid was modeled as a Hershel-Bulkley-Quemada plastic [45,46], permeability was modeled through the Kozeny-Carman relation, and relative permeabilities and capillary pressures where calculated through the van Genuchten model [13]. The Herschel-Bulkley-Quemada and Kozeny-Carman models couple the solid's rheology and absolute permeability to its porosity, making the solid harder to deform as its compressed and easier to flow through as it expands (and vice versa). Porosity was initialized as a normally distributed field.

The resulting simulations were implemented in OpenFOAM using a 500 by 500 grid with constant flow boundary conditions at the inlet, zero-gradient flow conditions at the outlet, and no-slip boundary conditions for solid displacement at all boundaries. To allow for a proper comparison between 2D simulations and 3D experiments in a Hele-Shaw cell of thickness $a$, an additional drag term $\left(12 \mu a^{-2} U_{f}\right)$ was included in the fluid momentum equation [47]. Last, the boundary effects of sliding friction and vertical confinement on the solid were neglected, a reasonable simplification that becomes significant at relatively low initial packing fractions $\left(\phi_{s} \sim 0.35\right)$ or high confining pressures [26]. If necessary, these effects could potentially be included into the model by including an additional drag term and a confining pressure into Eq. (5) as done in Ref. [18].

Further details regarding the base implementation of the model can be found in Ref. [36], the accompanying code [42], and Appendix B. The only major differences relative to our previous simulations are that we now include capillary effects and represent viscously stable drainage as opposed to imbibition (i.e., the injected glycerin is now nonwetting to the porous medium). A representative sample of the more than 400 simulated cases is presented in the phase diagrams shown in Fig. 3.

Overall, the results make intuitive sense. Figure 3(a) shows that, ceteris-paribus, less permeable solids are more prone to fracturing. This is due to the fact that, given a constant flow rate, lower permeability solids experience greater drag forces (see Darcy's Law). As described in similar studies [28], our results also show that solids with lower plastic yield stresses fracture more readily, as their solid structure cannot withstand the effects of relatively large viscous or capillary forces. The 

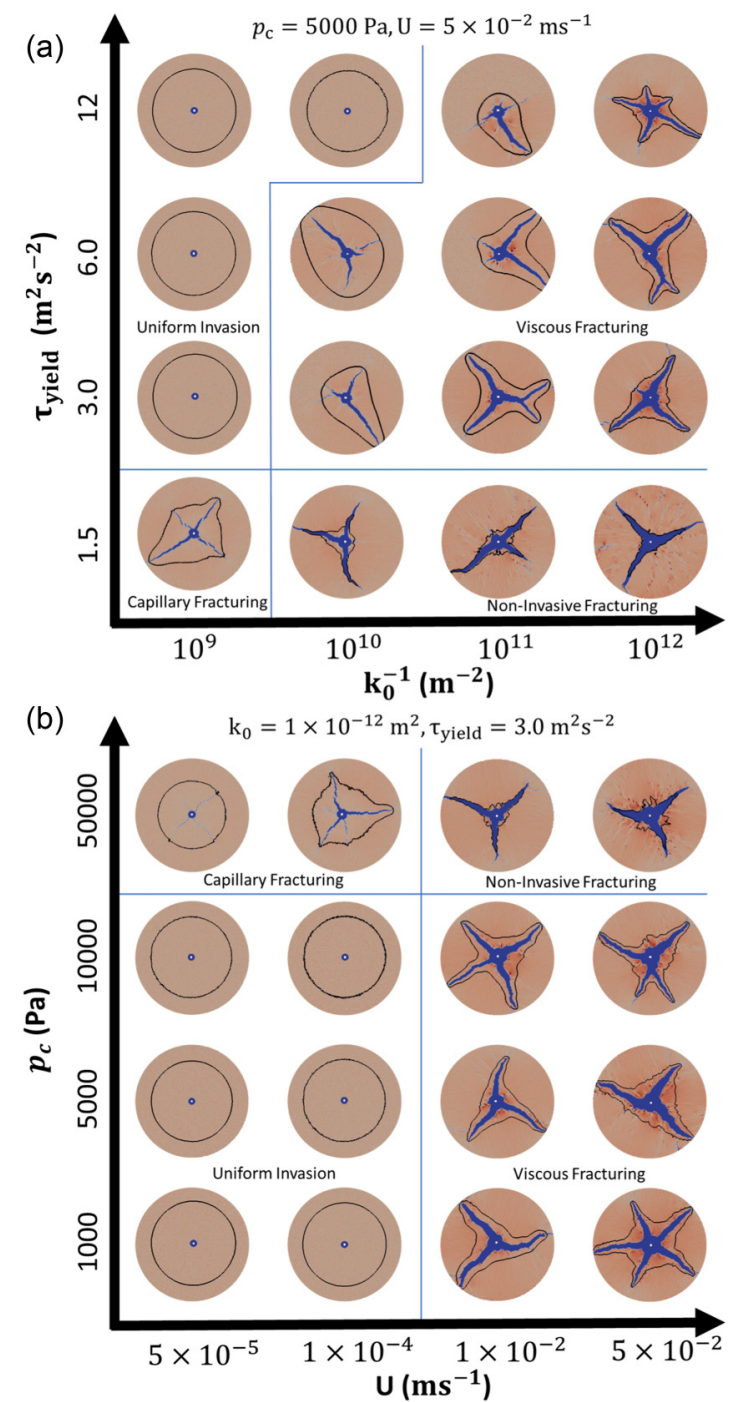

FIG. 3. Phase diagrams describing the effects of varying permeability, plastic yield stress, fluid injection rate, and capillary entry pressure on the transition from fluid drainage to fracturing. All cases are at $\phi_{s}=0.60 \pm 0.05$ and $\mu_{n}=5 \mathrm{cP}$. The remaining parameters are case-specific and can be found in each figure's upper legend. The areas separated by thin blue lines highlight and label the four deformation regimes described in Sec. IV. The color scheme is the same as in Fig. 2. Additional cases can be found in the Supplemental Materials [44]

$y$-axis behavior of Fig. 3(b) further shows that systems with higher entry pressures are more likely to fracture, i.e., the capillary stresses are more likely to overwhelm the solid's yield stress, in agreement with grain scale simulations [30]. Finally, Fig. 3(b) also shows that higher injection rates lead to more fracturing, as these induce a greater viscous drag on the solid structure.

\section{CHARACTERIZATION OF FRACTURING MECHANISMS}

The deformation regimes observed in the previous experiments can be delineated by two simple nondimensional parameters that quantify the balance between viscous pressure

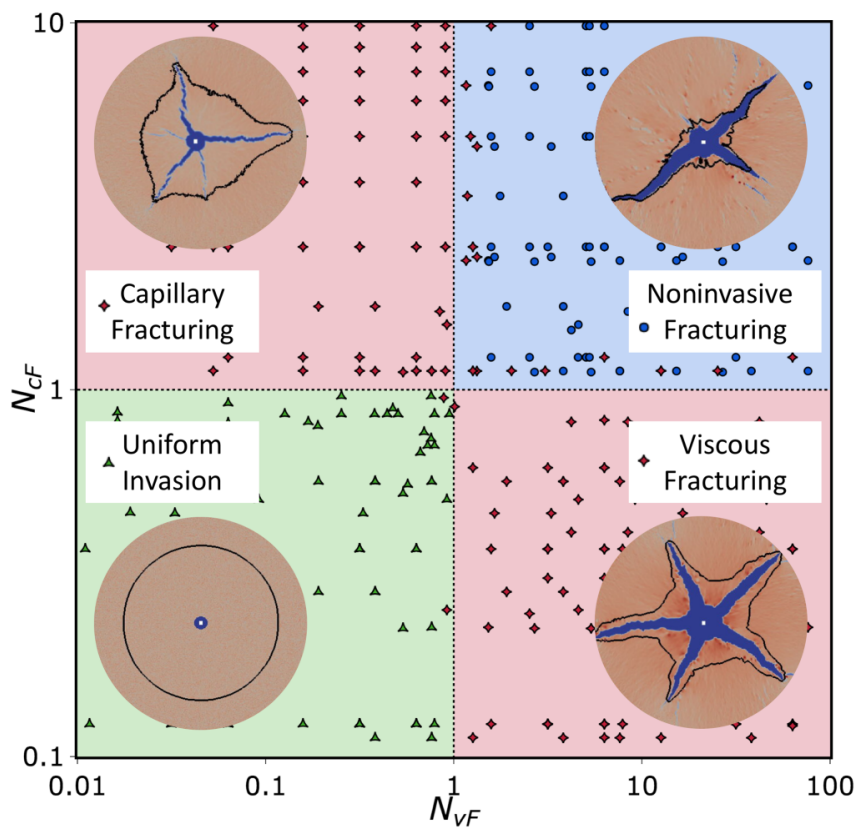

FIG. 4. Fluid invasion and fracturing in plastic porous media as a function of the viscous fracturing number $N_{\mathrm{vF}}$ and the capillary fracturing number $N_{\mathrm{cF}}$. The four image insets are representative samples of each fracturing regime, which are differentiated by the fractured-to-saturated area ratio $(R)$ : Green triangles denote uniform invasion $(R=0)$, red diamonds denote the invasive fracturing regimes $(0<R<0.75)$, and blue circles denote noninvasive fracturing $(R>0.75)$.

drop, solid softness, and capillary entry pressure.

$$
\begin{gathered}
N_{\mathrm{vF}}=\frac{\Delta p}{\tau_{\text {yield }} \rho_{s}}=\frac{\mu U r_{\text {in }}}{k \tau_{\text {yield }} \rho_{s}} \ln \left(\frac{r_{\text {out }}}{r_{\text {in }}}\right), \\
N_{\mathrm{cF}}=\frac{p_{c, 0}}{\tau_{\text {yield }} \rho_{s}}=\frac{2 \gamma}{r_{\text {pore }} \tau_{\text {yield }} \rho_{s}} .
\end{gathered}
$$

Here, the viscous fracturing number $\left(N_{\mathrm{vF}}\right)$ represents the ratio between the viscous pressure drop and the solid's structural forces. It embodies the question: Does fluid flow generate sufficient friction to induce fracturing? As shown in Fig. 4, the answer is no if $N_{\mathrm{vF}}<1$ and yes if $N_{\mathrm{vF}}>1$. At high capillary numbers, this number is equivalent to the fracturing number presented by Holtzman et al. [29] for granular solids and is closely related to the "dimensionless time" used to analyze fracture propagation in Huang et al. [48]. It also conforms with the experimental finding by Zhou et al. [49] that fracture initiation is only a function of the resulting fluid pressure drop, irrespective of the injection rate or fluid viscosity used to create it. Furthermore, it illustrates why increasing the injection rate and decreasing the permeability have similar effects in Fig. 3.

Complementarily, the capillary fracturing number $\left(N_{\mathrm{cF}}\right)$ represents the ratio between the capillary entry pressure and the solid's structural forces; it embodies the question: Does multiphase flow generate sufficient capillary stresses to fracture the solid? Figure 4 shows that when $N_{\mathrm{cF}}<1$ drainage is the preferential flow mechanism and when $N_{\mathrm{cF}}>1$ fracturing becomes the dominant phenomenon. The definition of 


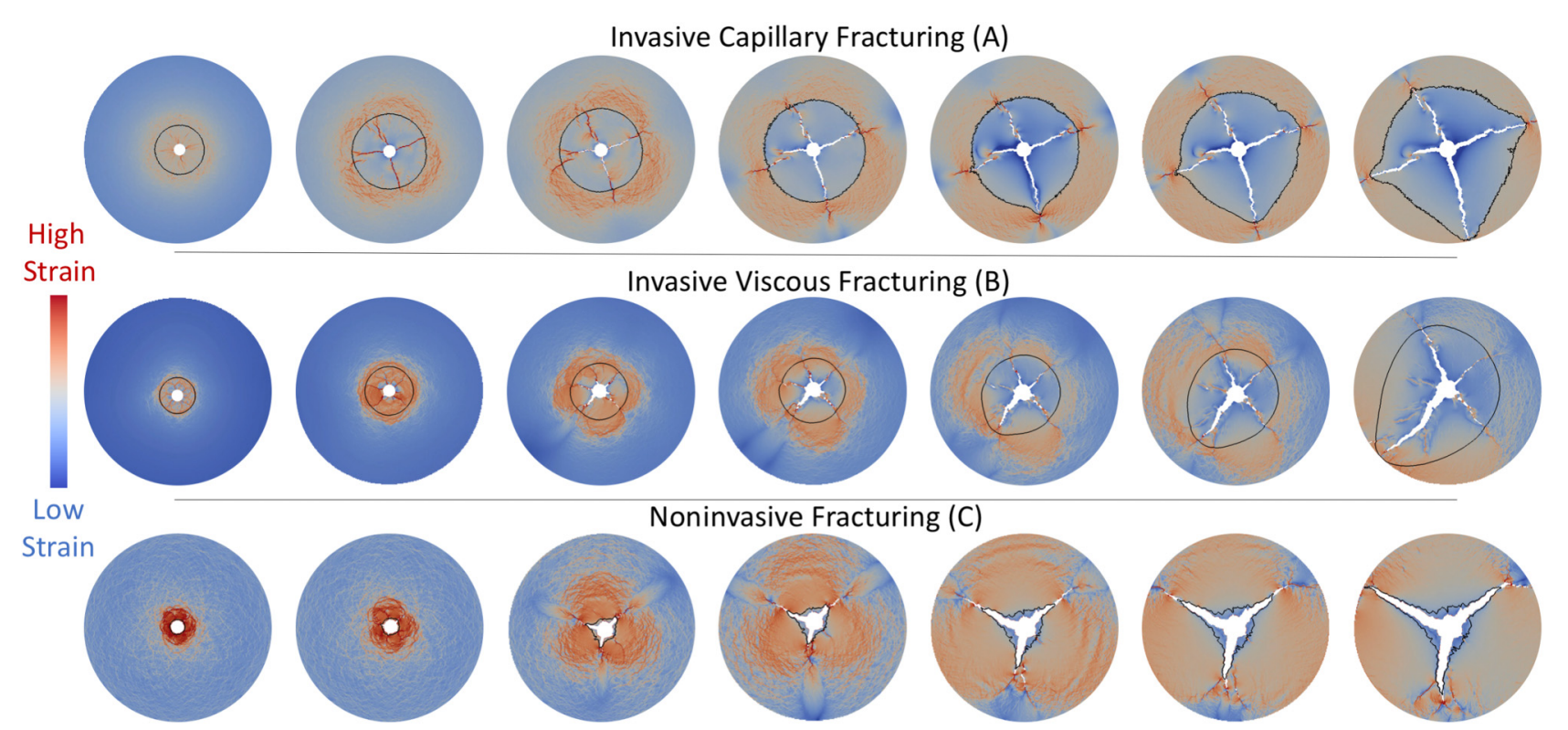

FIG. 5. Dynamic fracture formation mechanisms. Each row represents the time-dependent fracture formation process for each fracturing type, where time advances from left to right. Here, the red-blue color scheme represents the log-normalized strain-rate magnitude specific to each simulated case, fractures are shown in white and the advancing fluid-fluid interface is shown as a thin black line.

these fracturing numbers effectively decouples the effects that viscous and capillary forces have on fracturing mechanics. This is in contrast with previous studies, where fracturing was characterized as a function of the capillary number $(\mathrm{Ca} \sim$ $\left.N_{\mathrm{vF}} / N_{\mathrm{cF}}\right)$ and/or the sum of the two fracturing numbers $\left(N_{F} \sim\right.$ $\left.N_{\mathrm{cF}}+N_{\mathrm{cF}}\right)$ used here [28,29]. As described below, this subtle change in perspective allows us to define distinct material failure regimes that reflect individual changes in the magnitude of capillary and viscous forces, as opposed to their sum or relative magnitudes.

The previous analysis yields the rudimentary conclusion that fracturing should occur if either of the fracturing numbers is greater than unity, as confirmed by our simulations. However, our simulations and choice of fracturing numbers further demonstrate the existence of three distinct fracturing regimes (Figs. 3 and 4). The first regime, referred here as noninvasive fracturing $\left(N_{\mathrm{vF}}>1\right.$ and $\left.N_{\mathrm{cF}}>1\right)$, is characterized by fracturing of the porous solid with minimal fluid invasion, where fractures precede any invasion front. In the second regime, referred to here as invasive viscous fracturing $\left(N_{\mathrm{vF}}>1\right.$ and $N_{\mathrm{cF}}<1$ ), only the viscous stresses are sufficiently large to fracture the solid. This leads to the formation of relatively wide fractures enveloped and preceded by a nonuniform invasion front. Finally, in the third regime, referred to here as invasive capillary fracturing $\left(N_{\mathrm{vF}}<1\right.$ and $\left.N_{\mathrm{cF}}>1\right)$, only the capillary stresses are sufficiently large to fracture the solid. Given a constant injection rate, this leads to the formation of fractures preceded by an invasion front, as in the invasive viscous fracturing regime, but with a more uniform saturation front (due to lower viscous stresses) and less solid compaction (hence, narrower fractures). We note that the crossover between each of the four regimes is continuous, meaning that systems with $N_{\mathrm{vF}}$ or $N_{\mathrm{cF}} \sim 1$ can share elements of neighboring regimes.

Although $N_{\mathrm{vF}}$ and $N_{\mathrm{cF}}$ are fairly intuitive numbers, their impacts on fracture propagation mechanisms are not. For this reason, we also studied the dynamics of fracture nucleation and growth and the evolution of the solid's strain for all three fracturing regimes. As seen in Fig. 5, fracturing in the two invasive fracturing regimes is characterized by the initial formation of non-flow-bearing failure zones (hereafter referred to as cracks), which function as nucleation sites for the propagation of flow-bearing fractures, in agreement with conventional theory $[27,50]$. These cracks are formed by the simultaneous movement of large contiguous sections of the porous medium in different directions, a process induced by uniform fluid invasion into the porous medium. However, the similarities between both invasive fracturing zones end here. In the invasive viscous fracturing regime, fractures quickly become the dominant deformation mechanism, localizing the majority of the stresses and solid compaction around the advancing fracture tip. Conversely, in the invasive capillary fracturing regime, fluid-invasion continues to serve as the main flow mechanism and source of deformation, where fractures and cracks are slowly expanded due to the more evenly distributed capillarity-induced stresses localized at the advancing invasion front. Finally, noninvasive fracturing follows a different process, where there is little-to-no crack formation and fracture propagation is the main source of deformation and flow. Here, the co-advancing fracture and saturation fronts uniformly compress the solid around and in front of them until this deformation reaches the outer boundary of the simulated system [see the "jet" like-structures at fracture tips in Fig. 5(c)]. Pressure profiles and videos that further showcase these behaviours can be found in Appendix C and the Supplemental Material [44], respectively.

\section{INFLUENCE OF LOCALIZED AND UNIFORM DEFORMATION}

So far we have explored how independently changing $k, p_{c}$, and $\tau_{\text {yield }}$ (among others) can affect the fracturing of plastic 

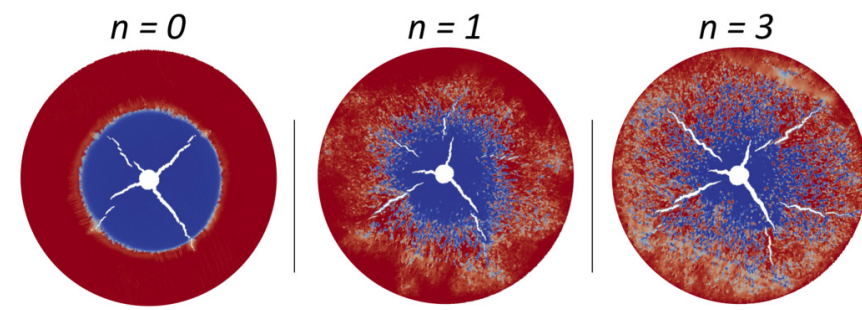

FIG. 6. Influence of the $\phi_{f}$-dependence of $p_{c}$ on fluid invasion (red and blue) and fracturing patterns (white) in the invasive capillary fracturing regime. Here, $n$ represents the sensitivity parameter in the Leverett J-function analog presented above.

materials. However, our results also have implications for situations in which these variables are all varied simultaneously, such as during the consolidation or compaction of soils, sediments, or viscoplastic sedimentary rocks (i.e., mudstones or clay-shales) [51,52]. In such situations, with increasing compaction, $k^{-1}, p_{c}$, and $\tau_{\text {yield }}$ should all increase, although at different rates. As such, we now study the effects of local and uniform deformation on the outlined fracturing regimes.

\section{A. Localized deformation}

The simulations presented above were carried out using the simplifying assumption that $p_{c}$ is invariant with $\phi_{f}$ (whereas $k$ and $\tau_{\text {yield }}$ are not). To evaluate the impact of this simplification on the results shown in Figs. 3 and 4, we carried out additional simulations for all four regimes with a deformation-dependent capillary entry pressure based on a simplified form of the Leverett $\mathrm{J}$-function where $p_{c, 0}=p_{c, 0}^{*}\left(\phi_{s} / \phi_{s}^{\text {avg }}\right)^{n}, p_{c, 0}^{*}$ is the capillary pressure at $\phi_{s}=\phi_{s}^{\text {avg }}$, and $n>0$ is a sensitivity parameter [53,54]. The results show that nonzero values of $n$ promote the creation of finger-like instabilities and the nucleation of cracks at the fluid invasion front, particularly in the invasive capillary fracturing regime. Simulation predictions with different $n$ values are shown in Fig. 6 in the invasive capillary fracturing regime and in the Supplemental Material [44] in other regimes.

Despite the additional complexity of the resulting fluid invasion and fracturing patterns, results with $n>0$ conform to the overall phase diagram presented in Fig. 4 . The results at $n=0$ are therefore highlighted in the previous sections due to the greater simplicity of their fluid and solid distribution patterns.

\section{B. Uniform deformation}

Having verified that the applicability of the fracturing numbers holds for systems were $k, \tau_{\text {yield }}$, and $p_{c}$ all vary with $\phi_{f}$, we now examine the effects of uniform compaction on said numbers. A direct analysis using the widely used porosity-parameter relationships implemented above (the Kozeny-Carman relation for $k$, Leverett J-Function for $p_{c}$, and Quemada model for $\left.\tau_{\text {yield }}[45,46,53]\right)$ yields the following fracturing number porosity-dependence:

$$
\begin{aligned}
& N_{\mathrm{vF}} \propto \frac{\left(1-\phi_{f}\right)^{2-D}\left(1-\phi_{f, \min } / \phi_{f}\right)}{\phi_{f}^{2}}, \\
& N_{\mathrm{cF}} \propto\left(1-\phi_{f}\right)^{2-D}\left(1-\phi_{f, \min } / \phi_{f}\right),
\end{aligned}
$$
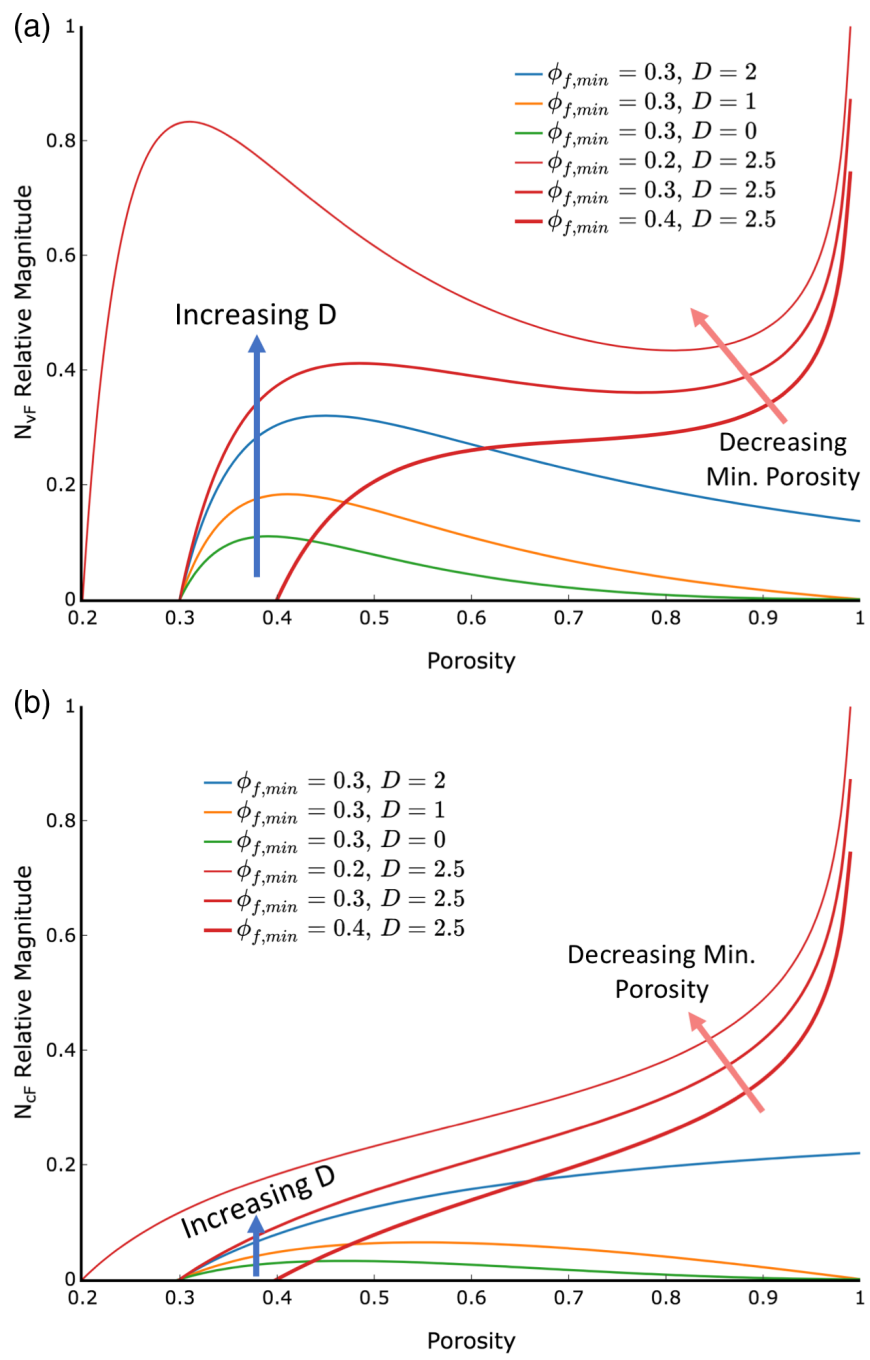

FIG. 7. Relative magnitude of the fracturing numbers $N_{\mathrm{vF}}$ (a) and $N_{\mathrm{cF}}$ (b), plotted as a function of porosity. Results are shown for different values of the fractal parameter $D$ and the minimum porosity parameter $\phi_{f, \text { min }}$ in the rheology model. Green, orange, blue, and red curves represent increasing values of $D$ if $\phi_{f, \min }=0.3$; increasingly thicker curves represent increasing values of $\phi_{f, \min }$ if $D=2.5$.

where $D$ is a rheological parameter based on the solid's fractal dimension (common values range for 1.7-2.9 for different clayey sediments [45]) and $\phi_{f, \min }$ is the minimum achievable porosity (i.e the maximum possible degree of compaction). Through these relations, we can see that uniform compaction (or expansion) can have a highly non-linear effect on fracturing. Equations (9) and (10) indicate that whereas $N_{\mathrm{cF}}$ tends to consistently decrease with increasing compaction, $N_{\mathrm{vF}}$ is considerably more susceptible to changes in $\phi_{f}$ and exhibits several changes in the sign of its first derivative when $D>2$, nonintuitively suggesting that fracturing can be either induced or suppressed through uniform compression. Plots of $N_{\mathrm{vF}}$ and $N_{\mathrm{cF}}$ as a function of solid fraction are reported in Fig. 7.

\section{CONCLUSIONS}

In this article, we used the multiphase DBB framework to create a phase diagram based on two nondimensional 
parameters that categorize the crossover between viscously stable fluid drainage and fracturing as a function of wettability, solid deformability, and hydrodynamics. To the best of our knowledge, our results are the first to simultaneously relate and vary all three of these properties to characterize multiphase flow in viscoplastic porous media. In agreement with previous studies, we observe that fracturing occurs if the viscous and/or capillary stresses are sufficient to overcome the solid's structural forces. Thus, when it comes to systems with multiple fluids, it is necessary to consider the effects of surface tension, wettability, and pore size on the fluids' propensity to fracture or invade the permeable solid. Furthermore, we found that the two nondimensional fracturing numbers described above delineate the existence of three fracturing regimes with distinct fracture propagation mechanisms. Last, we examined how uniform compression or expansion affect said nondimensional numbers and a system's propensity to fracture.

\section{ACKNOWLEDGMENTS}

This work was supported by the National Science Foundation under Grant No. EAR-1752982. F.J.C. acknowledges additional support from the Mary and Randall Hack 69 Fellowship of the High Meadows Environmental Institute at Princeton University.

\section{APPENDIX A: DESCRIPTION OF MULTISCALE PARAMETERS}

The closed-form expressions of the multiscale parameters $\mu k^{-1}, \boldsymbol{F}_{c}$, and $\boldsymbol{U}_{r}$ now follow. These are defined differently in porous $\left(\phi_{f}<1\right)$ and solid-free $\left(\phi_{f}=1\right)$ regions. A full derivation and discussion of these parameters can be found in Refs. [36,40].

$$
\begin{gathered}
\mu k^{-1}= \begin{cases}0 & \phi_{f}=1, \\
k_{0}^{-1}\left(\frac{k_{r, w}}{\mu_{w}}+\frac{k_{r, n}}{\mu_{n}}\right)^{-1} & \phi_{f}<1,\end{cases} \\
\boldsymbol{F}_{c}= \begin{cases}-\frac{\gamma}{\phi_{f}} \nabla \cdot\left(\boldsymbol{n}_{w, n}\right) \nabla \alpha_{w} & \phi_{f}=1, \\
M^{-1}\left(M_{w} \alpha_{n}-M_{n} \alpha_{w}\right) & \phi_{f}<1, \\
*\left(\nabla p_{c}+\left(\rho_{w}-\rho_{n}\right) \boldsymbol{g}\right) & \phi_{f}=1,\end{cases} \\
\boldsymbol{n}_{w, n}=\left\{\begin{array}{cc}
\frac{\nabla \alpha_{w}}{\left|\nabla \alpha_{w}\right|} & \phi_{f}=1, \\
\cos (\theta) \boldsymbol{n}_{\mathrm{wall}}+\sin (\theta) \boldsymbol{t}_{\mathrm{wall}} & \text { at } \zeta,
\end{array}\right. \\
\boldsymbol{U}_{r}=\left\{\begin{array}{c}
C_{\alpha} \max \left(\left|\boldsymbol{U}_{f}\right|\right) \frac{\nabla \alpha_{w}}{\left|\nabla \alpha_{w}\right|}, \\
-\left(\frac{M_{w}}{\alpha_{w}}-\frac{M_{n}}{\alpha_{n}}\right) \nabla p \\
+\left(\frac{\rho_{w} M_{w}}{\alpha_{w}}-\frac{\rho_{n} M_{n}}{\alpha_{n}}\right) \boldsymbol{g} \\
+\left(\frac{M_{w} \alpha_{n}}{\alpha_{w}}+\frac{M_{n} \alpha_{w}}{\alpha_{n}}\right) \nabla p_{c} \\
-\left(\frac{M_{w}}{\alpha_{w}}-\frac{M_{n}}{\alpha_{n}}\right) p_{c} \nabla \alpha_{w}
\end{array}\right]
\end{gathered}
$$

where $C_{\alpha}$ is an interface compression parameter (traditionally set to values between 1 and 4 in the volume-of-fluid method), $\zeta$ is the interface between the porous and solid-free regions, $k_{0}$ is the absolute permeability, $k_{r, i}$ and $M_{i}=k_{0} k_{i, r} / \mu_{i}$ are the relative permeability and mobility of each fluid, and $M=$
$M_{w}+M_{n}$. Last, $\theta$ is the imposed contact angle at the porous wall, and $\boldsymbol{n}_{\text {wall }}$ and $\boldsymbol{t}_{\text {wall }}$ are the normal and tangential directions relative to said wall, respectively.

\section{APPENDIX B: GENERAL METHODOLOGY FOR THE FRACTURING SIMULATIONS SHOWN IN FIG. 2}

As shown in our previous work [36], the simulations shown in Fig. 2 were implemented as follows: Numerical parameters were set to the known properties of aqueous glycerin, air, and sand $\left(\rho_{\text {gly }}=1250 \mathrm{~kg} / \mathrm{m}^{3}, \mu_{\text {gly }}=5\right.$ to $176 \mathrm{cP}$, $\rho_{\text {air }}=1 \mathrm{~kg} / \mathrm{m}^{3}, \mu_{\text {air }}=0.017 \mathrm{cP}, \rho_{s}=2650 \mathrm{~kg} / \mathrm{m}^{3}$ ), the airglycerin surface tension $\gamma=0.063 \mathrm{~kg} / \mathrm{s}^{2}$, and the sand grain radius $r_{s}=100 \mu \mathrm{m}$. To mimic the existence of sub-REV scale heterogeneity, the solid fraction was initialized as a normally distributed field $\phi_{s}=0.64 \pm 0.05$. To account for the nonreversible and compressive nature of the experiments, the deformable solid was modeled as a Hershel-BulkleyQuemada plastic with a density-normalized yield stress of $\tau_{\text {yield }}=16.02 \mathrm{~m}^{2} / \mathrm{s}^{2}[36,55,56]$. Permeability was modeled as a function of porosity through the Kozeny-Carman relation: $k=k_{0} \phi_{f}^{3} \phi_{s}^{-2}$ with $k_{0}=6.7 \times 10^{-12} \mathrm{~m}^{2}$. Relative permeabilities were calculated through the Van Genuchten model [13] with wettability parameter $m=0.99$; capillary effects were assumed negligible.

\section{APPENDIX C: FRACTURING PRESSURE PROFILES}

Figure 8 shows representative normalized pressure profiles for all four deformation regimes. Note how the two invasive fracturing regimes exhibit a combination of the characteristic behavior shown in the uniform invasion and noninvasive fracturing pressure curves. Abrupt pressure drops and gradual pressure decreases correspond, respectively, to the crack nucleation and fracture propagation phenomena discussed in the text.

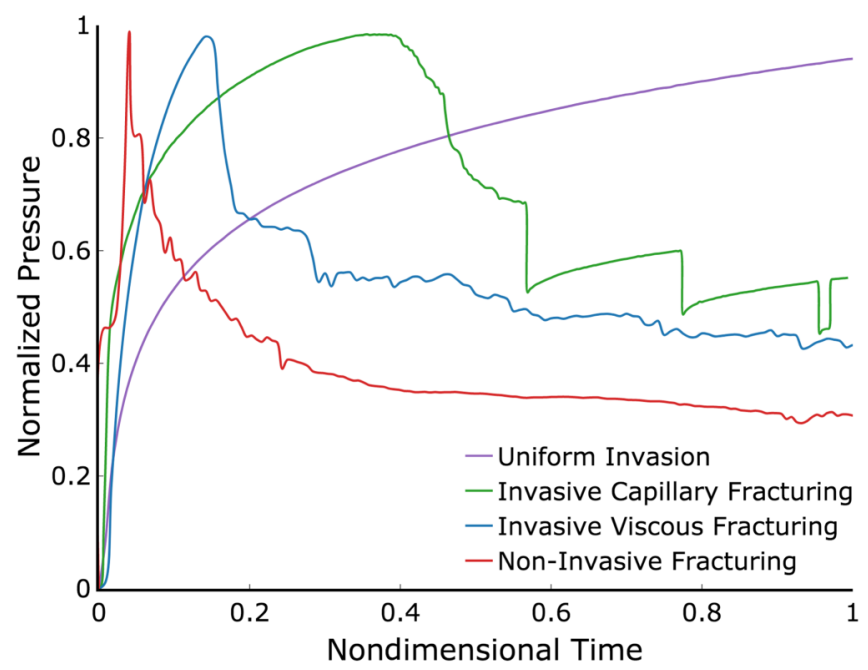

FIG. 8. Representative normalized pressure profiles for all four fracturing regimes. All pressure profiles where normalized by the maximum pressure achieved by each simulation and by the time it took for the fluid-fluid interface to reach the outer boundary in each simulation. 


\section{APPENDIX D: SOLID RHEOLOGY MODELS}

\section{Hershel-Bulkley plasticity}

A Bingham plastic is a material that deforms only once it is under a sufficiently high stress. After this yield stress is reached, it will deform viscously and irreversibly. The Herschel-Bulkley rheological model combines the properties of a Bingham plastic with a power-law viscosity model, such that said plastic can be shear thinning or shear thickening during deformation. In OpenFOAM this model is implemented as follows:

$$
\boldsymbol{\sigma}=\mu_{s}^{\mathrm{eff}}\left(\boldsymbol{\nabla} \boldsymbol{U}_{s}+\left(\boldsymbol{\nabla} \boldsymbol{U}_{s}\right)^{T}-\frac{2}{3} \boldsymbol{\nabla} \cdot\left(\boldsymbol{U}_{s} \boldsymbol{I}\right)\right),
$$

where $\mu_{s}^{\text {eff }}$ is the effective solid plastic viscosity, which is then modeled through a power-law expression:

$$
\mu_{s}^{\text {eff }}=\min \left(\mu_{s}^{0}, \frac{\tau_{\text {yield }}}{\eta}+\mu_{s} \eta^{n-1}\right),
$$

where $\mu_{s}^{0}$ is the limiting viscosity (set to a large value), $\tau_{\text {yield }}$ is the yield stress, $\mu_{s}$ is the viscosity of the solid once the yield stress is overcome, $n$ is the flow index ( $n=1$ for constant viscosity), and $\eta$ is the shear rate.

\section{Quemada rheology model}

The Quemada rheology model $[45,46]$ is a simple model that accounts for the fact that the average yield stress and effective viscosity of a plastic are functions of the solid fraction. These two quantities are large at high solid fractions and small at low solid fractions, as described by the following relations:

$$
\begin{gathered}
\tau_{\text {yield }}=\tau_{0}\left(\frac{\left(\phi_{s} / \phi_{s}^{\max }\right)}{\left(1-\phi_{s} / \phi_{s}^{\max }\right)}\right)^{D}, \\
\mu_{s}=\frac{\mu_{0}}{\left(1-\frac{\phi_{s}}{\phi_{s}^{\max }}\right)^{2}},
\end{gathered}
$$

here, $\phi_{s}^{\max }$ is the maximum solid fraction possible (perfect incompressible packing), $\tau_{0}$ is the yield stress at $\phi_{s}=$ $\phi_{s}^{\max } / 2, \mu_{0}$ is the viscosity of the fluid where the solid would be suspended at low solid fractions (high fluid fractions), and $D$ is a scaling parameter based on the solid's fractal dimension.

\section{APPENDIX E: NOMENCLATURE}

$n \quad$ Leverett $J$-function sensitivity parameter

$\alpha_{n} \quad$ Saturation of the nonwetting phase

$\alpha_{w} \quad$ Saturation of the wetting phase

$\sigma \quad$ Elastic or plastic solid stress tensor $(\mathrm{Pa})$

$F_{c} \quad$ Surface tension force $\left(\mathrm{Pam}^{-1}\right)$

$g \quad$ Gravity vector $\left(\mathrm{ms}^{-2}\right)$

$n_{\text {wall }} \quad$ Normal vector to the porous surface

$S \quad$ Single-field fluid viscous stress tensor $(\mathrm{Pa})$

$t_{\text {wall }} \quad$ Tangent vector to the porous surface

$U_{f} \quad$ Single-field fluid velocity $\left(\mathrm{ms}^{-1}\right)$

$U_{r} \quad$ Relative fluid velocity $\left(\mathrm{ms}^{-1}\right)$

$U_{s} \quad$ Solid velocity $\left(\mathrm{ms}^{-1}\right)$

$\gamma \quad$ Fluid-fluid interfacial tension (Pam)

$\mu_{i} \quad$ Viscosity of phase $i$ (Pas)

$\phi_{f} \quad$ Porosity field

$\phi_{s} \quad$ Solid fraction field

$\rho_{f} \quad$ Single-field fluid density $\left(\mathrm{kgm}^{-3}\right)$

$\rho_{i} \quad$ Density of phase $i\left(\mathrm{kgm}^{-3}\right)$

$\tau_{0} \quad$ Plastic yield stress $(\mathrm{Pa})$

$\theta \quad$ Surface contact angle

$C_{\alpha} \quad$ Parameter for the compression velocity model

$D \quad$ Solid Fractal Rheological Parameter

$k \quad$ Apparent permeability $\left(\mathrm{m}^{2}\right)$

$k_{0} \quad$ Absolute permeability $\left(\mathrm{m}^{2}\right)$

$k_{r, i} \quad$ Relative permeability with respect to phase $i$

$M \quad$ Total mobility $\left(\mathrm{kg}^{-1} \mathrm{~m}^{3} \mathrm{~s}^{-1}\right)$

$M_{i} \quad$ Mobility of phase i $\left(\mathrm{kg}^{-1} \mathrm{~m}^{3} \mathrm{~s}^{-1}\right)$

$p \quad$ Single-field fluid pressure $(\mathrm{Pa})$

$p_{c} \quad$ Capillary pressure $(\mathrm{Pa})$

$p_{c, 0} \quad$ Entry capillary pressure $(\mathrm{Pa})$
[1] C. Bächer and S. Gekle, Computational modeling of active deformable membranes embedded in three-dimensional flows, Phys. Rev. E 99, 062418 (2019).

[2] L. Räss, N. S. Simon, and Y. Y. Podladchikov, Spontaneous formation of fluid escape pipes from subsurface reservoirs, Sci. Rep. 8, 11116 (2018).

[3] G. D. Towner, The mechanics of cracking of drying clay, J. Agric. Eng. Res. 36, 115 (1987).

[4] R. Lenormand, Pattern growth and fluid displacements through porous media, Physica A 140, 114 (1986).

[5] K. J. Måløy, J. Feder, and T. Jøssang, Viscous Fingering Fractals in Porous Media, Phys. Rev. Lett. 55, 2688 (1985).

[6] P. G. Saffman and G. I. Taylor, The penetration of a fluid into a porous medium or Hele-Shaw cell containing a more viscous liquid, Proc. R. Soc., Ser. A 245, 312 (1958).

[7] J. P. Stokes, D. A. Weitz, J. P. Gollub, A. Dougherty, M. O. Robbins, P. M. Chaikin, and H. M. Lindsay, Interfacial Stability of Immiscible Displacement in a Porous Medium, Phys. Rev. Lett. 57, 1718 (1986).

[8] M. Ferer, C. Ji, G. S. Bromhal, J. Cook, G. Ahmadi, and D. H. Smith, Crossover from capillary fingering to viscous fingering for immiscible unstable flow: Experiment and modeling, Phys. Rev. E 70, 016303 (2004).

[9] Y. C. Yortsos, B. Xu, and D. Salin, Phase Diagram of Fully Developed Drainage in Porous Media, Phys. Rev. Lett. 79, 4581 (1997).

[10] D. Picchi and I. Battiato, The impact of pore-scale flow regimes on upscaling of immiscible two-phase flow in porous media, Water Resour. Res. 54, 6683 (2018).

[11] D. Picchi and I. Battiato, Relative permeability scaling from pore-scale flow regimes, Water Resour. Res. 55, 3215 (2019).

[12] R. H. Brooks and A. T. Corey, Hydraulic properties of porous media, Hydrol. Papers, Colorado State University 3, 37 (1964). 
[13] M. T. van Genuchten, Closed-form equation for predicting the hydraulic conductivity of unsaturated soils, Soil Sci. Soc. Am. J. 44, 892 (1980).

[14] M. A. Biot, General theory of three-dimensional consolidation, J. Appl. Phys. 12, 155 (1941).

[15] K. Terzaghi, Theoretical Soil Mechanics (John Wiley \& Sons, Hoboken, NJ, 1943).

[16] L. C. Auton and C. W. MacMinn, From arteries to boreholes: Steady-state response of a poroelastic cylinder to fluid injection, Proc. R. Soc. A 473, 20160753 (2017).

[17] T. Bertrand, J. Peixinho, S. Mukhopadhyay, and C. W. MacMinn, Dynamics of swelling and drying in a spherical gel, Phys. Rev. Appl. 6, 064010 (2016).

[18] F. J. Carrillo and I. C. Bourg, A Darcy-Brinkman-Biot approach to modeling the hydrology and mechanics of porous media containing macropores and deformable microporous regions, Water Resour. Res. 55, 8096 (2019).

[19] C. W. MacMinn, E. R. Dufresne, and J. S. Wettlaufer, Fluiddriven deformation of a soft granular material, Phys. Rev. X 5, 011020 (2015).

[20] A. Al-Busaidi, J. F. Hazzard, and R. P. Young, Distinct element modeling of hydraulically fractured Lac du Bonnet granite, J. Geophys. Res.: Solid Earth 110, B06302 (2005).

[21] L. Li and R. M. Holt, Simulation of flow in sandstone with fluid coupled particle model, DC Rocks 2001 Proceedings of the 38th U.S. Symposium on Rock Mechanics (USRMS), 165 (2001).

[22] Z. Lei, E. Rougier, A. Munjiza, H. Viswanathan, and E. E. Knight, Simulation of discrete cracks driven by nearly incompressible fluid via 2D combined finite-discrete element method, Int. J. Numer. Anal. Methods Geomech. 43, 1724 (2019).

[23] H. J. Cho, N. B. Lu, M. P. Howard, R. A. Adams, and S. S. Datta, Crack formation and self-closing in shrinkable, granular packings, Soft Matter 15, 4689 (2019).

[24] M. Prodanović, J. T. Holder, and S. L. Bryant, Pore scale coupling of fluid displacement and unconsolidated sediment mechanics, Int. J. Oil, Gas Coal Technol. 5, 157 (2012).

[25] J. M. Campbell, D. Ozturk, and B. Sandnes, Gas-driven fracturing of saturated granular media, Phys. Rev. Appl. 8, 064029 (2017).

[26] B. Sandnes, E. G. Flekkøy, H. A. Knudsen, K. J. Måløy, and H. See, Patterns and flow in frictional fluid dynamics, Nat. Commun. 2, 288 (2011).

[27] H. Shimizu, S. Murata, and T. Ishida, The distinct element analysis for hydraulic fracturing in hard rock considering fluid viscosity and particle size distribution, Int. J. Rock Mech. Min. Sci. 48, 712 (2011).

[28] R. Holtzman and R. Juanes, Crossover from fingering to fracturing in deformable disordered media, Phys. Rev. E 82, 046305 (2010).

[29] R. Holtzman, M. L. Szulczewski, and R. Juanes, Capillary Fracturing in Granular Media, Phys. Rev. Lett. 108, 264504 (2012).

[30] A. K. Jain and R. Juanes, Preferential mode of gas invasion in sediments: Grain-scale mechanistic model of coupled multiphase fluid flow and sediment mechanics, J. Geophys. Res.: Solid Earth 114, B08101 (2009).

[31] Y. Meng, B. K. Primkulov, Z. Yang, C. Y. Kwok, and R. Juanes, Jamming transition and emergence of fracturing in wet granular media, Phys. Rev. Res. 2, 022012(R) (2020).
[32] W. Abdallah, J. S. Buckley, A. Carnegie, J. Edwards, B. Herold, A. Graue, T. Habashy, S. Nikita, S. Claude, H. Hussain, M. Bernard, and Z. Murtaza, Fundamentals of wettability, Oilfield Rev. 44 (2007).

[33] A. M. Alhammadi, A. Alratrout, K. Singh, B. Bijeljic, and M. J. Blunt, In situ characterization of mixed-wettability in a reservoir rock at subsurface conditions, Sci. Rep. 7, 10753 (2017).

[34] A. AlRatrout, M. J. Blunt, and B. Bijeljic, Wettability in complex porous materials, the mixed-wet state, and its relationship to surface roughness, Proc. Nat. Acad. Sci. U.S.A. 115, 8901 (2018).

[35] M. Arif, Y. Zhang, and S. Iglauer, Shale wettability: Data sets, challenges, and outlook, Energy Fuels 35, 2965 (2021).

[36] F. J. Carrillo and I. C. Bourg, Modeling multiphase flow within and around deformable porous materials: A Darcy-BrinkmanBiot approach, Water Resour. Res. 57, e2020WR028734 (2021).

[37] C. W. Hirt and B. D. Nichols, Volume of fluid (VOF) method for the dynamics of free boundaries, J. Comput. Phys. 39, 201 (1981).

[38] B. Jha and R. Juanes, Coupled multiphase flow and poromechanics: A computational model of pore pressure effects on fault slip and earthquake triggering, Water Resour. Res. 50, 3776 (2014).

[39] J. Kim, H. A. Tchelepi, and R. Juanes, Rigorous coupling of geomechanics and multiphase flow with strong capillarity, SPE J. 18, 1123 (2013).

[40] F. J. Carrillo, I. C. Bourg, and C. Soulaine, Multiphase flow modeling in multiscale porous media: An open-source microcontinuum approach, J. Comput. Phys. X 8, 100073 (2020).

[41] S. Whitaker, Flow in porous media I: A theoretical derivation of Darcy's law, Transp. Porous Media 1, 3 (1986).

[42] F. J. Carrillo and I. C. Bourg, hybridBiotInterFoam (2020), https://github.com/Franjcf.

[43] H. Huang, F. Zhang, P. Callahan, and J. A. Ayoub, Fluid injection experiments in 2D porous media, SPE J. 17, 903 (2012).

[44] See Supplemental Material at http://link.aps.org/supplemental/ 10.1103/PhysRevE.103.063106 for additional fracturing phasediagrams and figures showing the effects of nonuniform capillary pressure on the different fracturing-invasion regimes.

[45] J. Spearman, An examination of the rheology of flocculated clay suspensions, Ocean Dynam. 67, 485 (2017).

[46] D. Quemada, Rheology of concentrated disperse systems and minimum energy dissipation principle, Rheol. Acta 16, 82 (1977).

[47] A. Ferrari, J. Jimenez-Martinez, T. Le Borgne, Y. Méheust, and I. Lunati, Challenges in modeling unstable two-phase flow experiments in porous micromodels, Water Resour. Res. 51, 1381 (2015).

[48] H. Huang, F. Zhang, P. Callahan, and J. Ayoub, Granular Fingering in Fluid Injection Into Dense Granular Media in a Hele-Shaw Cell, Phys. Rev. Lett. 108, 258001 (2012).

[49] J. Zhou, Y. Dong, C. J. De Pater, and P. L. Zitha, Experimental study of the impact of shear dilation and fracture behavior during polymer injection for heavy oil recovery in unconsolidated reservoirs, in Proceedings of the Canadian Unconventional Resources and International Petroleum Conference of the 
Society of Petroleum Engineering (Calgary, Alberta, Canada, 2010).

[50] K. M. Hubbert and D. G. Willis, Mechanics of hydraulic fracturing, Trans. Soc. Petroleum Eng. AIME 210, 153 (1957).

[51] D. E. Daniel and Y.-K. Wu, Compacted clay liners and covers for arid sites, J. Geotech. Eng. 119, 223 (1993).

[52] J. F. Harrington, C. C. Graham, R. J. Cuss, and S. Norris, Gas network development in a precompacted bentonite experiment: Evidence of generation and evolution, Appl. Clay Sci. 147, 80 (2017).

[53] M. Leverett, Capillary behavior in porous solids, Trans. AIME 142, 152 (1941).
[54] B. Li and S. M. Benson, Influence of small-scale heterogeneity on upward $\mathrm{CO} 2$ plume migration in storage aquifers, Adv. Water Resour. 83, 389 (2015).

[55] A.-S. Ahmed, Z. Karim, W. Gay, M. Fanhong, and S. Manoj, Fracture propagation and formation disturbance during injection and frac-pack operations in soft compacting rocks, in Proceedings of the SPE Annual Technical Conference and Exhibition (Society of Petroleum Engineers, Dallas, TX, 2007), pp. 3453-3464.

[56] D. B. van Dam, P. Papanastasiou, and C. J. de Pater, Impact of rock plasticity on hydraulic fracture propagation and closure, SPE Prod. Facil. 17, 149 (2002). 